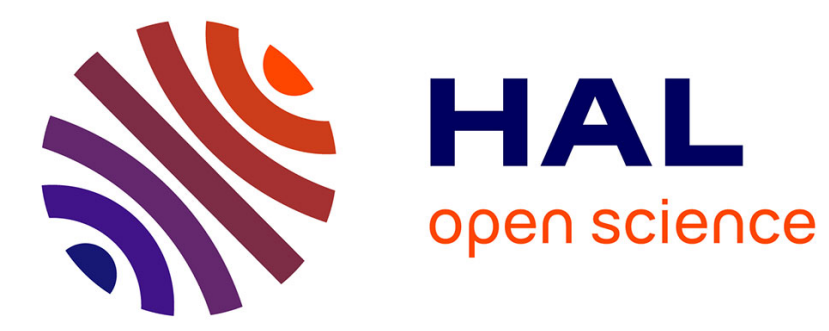

\title{
Design of observers implemented over FlexRay networks
}

Wei Wang, Dragan Nesic, Romain Postoyan

\section{To cite this version:}

Wei Wang, Dragan Nesic, Romain Postoyan. Design of observers implemented over FlexRay networks. Australian Control Conference, AUCC 2014, Nov 2014, Canberra, Australia. hal-01080094

\section{HAL Id: hal-01080094 https://hal.science/hal-01080094}

Submitted on 31 Dec 2014

HAL is a multi-disciplinary open access archive for the deposit and dissemination of scientific research documents, whether they are published or not. The documents may come from teaching and research institutions in France or abroad, or from public or private research centers.
L'archive ouverte pluridisciplinaire $\mathbf{H A L}$, est destinée au dépôt et à la diffusion de documents scientifiques de niveau recherche, publiés ou non, émanant des établissements d'enseignement et de recherche français ou étrangers, des laboratoires publics ou privés. 


\title{
Design of observers implemented over FlexRay networks
}

\author{
Wei Wang, Dragan Nešić and Romain Postoyan
}

\begin{abstract}
We investigate the observer design for nonlinear systems whose measurements are sent over a network governed by FlexRay. FlexRay is a communication protocol used in the automotive industry which has the feature to switch between two scheduling rules associated with the two segments of its communication cycles. The objective of this paper is to generalize existing works on emulated observers for networked control systems (NCS) to be applicable to NCS with FlexRay. We propose for that purpose a novel hybrid model and guarantee the observer convergence provided that, for each segment, the scheduling rules are uniformly globally exponentially stable and the maximal allowable transmission intervals satisfy given explicit bounds. The analysis relies on the use of an hybrid Lyapunov function we recently constructed to investigate the stabilization of NCS with FlexRay. We finally apply the approach to a class of globally Lipschitz systems, which includes linear time-invariant systems as a particular case.
\end{abstract}

\section{INTRODUCTION}

Networked control systems (NCS) are characterized by the use of a shared serial communication channel to connect spatially distributed sensors and actuators with the control unit. The communication links have some advantages but introduce communication constraints which may have a severe impact on the desired performance requirements. In this paper, we address the observer design problem for NCS and we concentrate on the effect of time-varying data sampling and scheduling. The feature of our work is that we consider FlexRay protocol [2], which switches between two scheduling rules. Because of these switches, related available results on the observer design for NCS in [9], [10], [11] are not applicable and we need to solve nontrivial issues in terms of modeling and analysis. Our motivation to study NCS with FlexRay is justified by the fact that this communication protocol is increasingly used in the automotive industry and estimation methods for such systems are currently missing in the literature. A few works on the other hand analyze stability of NCS with FlexRay, see [6], [12] for instance.

We design the observer using the emulation approach like in [9], [10], [11]. The basic idea is to first synthesize the observer while ignoring the communication constraints.

This work is supported by the Australian Research Council under the Discovery Project and Future Fellow program, the ANR under the grant COMPACS (ANR-13-BS03-0004-02) and the European 7th Framework Network of Excellence "Highly-complex and networked control systems" (HYCON2 No. 257462).

W. Wang and D. Nešić are with the Department of Electrical and Electronic Engineering, The University of Melbourne, Parkville, 3010, Victoria, Australia wei.wang@unimelb.edu.au, dnesic@unimelb.edu.au

R. Postoyan is with the Universite de Lorraine, CRAN, UMR 7039 and the CNRS, CRAN, UMR 7039, France romain.postoyan @univ-lorraine.fr
Then, the observer is implemented over the network and conditions on the latter are derived to preserve the convergence properties of the observer. The emulation approach is advantageous as it allows us to use various available observer design tools for continuous-time systems. As mentioned above, the related results in [9], [10], [11] cannot be used when considering protocols with switching scheduling rules such as FlexRay [2].

The problem is modeled as a hybrid system using the formalism of [3], for which a jump either describes a transmission or a segment switch. The proposed NCS model is different from the one in [12] because our target is estimation and not stabilization. We consider static and dynamic segments of any given lengths for which transmissions are governed by uniformly globally exponentially stable (UGES) protocols, which cover the round robin (RR) and the tryonce-discard (TOD) protocols as particular cases, see [7]. Employing a Lyapunov function we recently constructed to study stabilization of the NCS with FlexRay in [12], we ensure that the estimation error asymptotically converges to the origin provided the MATIs of each segment satisfy a given explicit bound and by appropriately emulating the observer. The obtained results are shown to be applicable to a class of globally Lipschitz systems which includes linear time invariant systems as a special case and for which these results are new.

The paper is organized as follows. We state the problem in Section II. The observer emulation is explained in Section III. The protocol model and the hybrid model for NCS are respectively presented in Sections IV and V. We study the convergence properties of the emulated observer in Section VI. A case study is given in Section VII. Conclusions and possible future works directions are provided in Section VIII.

\section{PRoblem StATEMENT}

Notation. Let $\mathbb{Z}_{>0}:=\{1,2, \cdots\}, \mathbb{Z}_{\geq 0}:=\{0,1,2, \cdots\}$ and $\mathbb{R}_{\geq 0}:=[0, \infty)$. The Euclidean norm of a vector or a matrix is denoted by $|\cdot|$ and $\lambda_{\min }(P), \lambda_{\max }(P)$ respectively stand for the minimum and maximum eigenvalues of a real symmetric positive definite matrix $P$. Given a closed set $\mathcal{A} \subset \mathbb{R}^{n}$ and $x \in \mathbb{R}^{n}$, we define the distance of a vector $x$ to $\mathcal{A}$ as $|x|_{\mathcal{A}}:=$ $\inf _{y \in \mathcal{A}}|x-y|$. A set-valued mapping $M: \mathbb{R}^{m} \rightrightarrows \mathbb{R}^{n}$ is outer semi-continuous (OSC) if and only if its graph $\{(y, z)$ : $\left.y \in \mathbb{R}^{m}, z \in M(y)\right\}$ is closed, see Lemma 5.10 in [3]. For $x \in \mathbb{R}^{n}$ and $y \in \mathbb{R}^{m}$, the notation $(x, y)$ denotes $\left[x^{T}, y^{T}\right]^{T}$.

\section{A. Objective}

Consider the nonlinear plant model 


$$
\dot{x}=\mathbf{f}(x) \quad y=\mathbf{g}(x),
$$

where $x \in \mathbb{R}^{n_{x}}$ is the state and $y \in \mathbb{R}^{n_{y}}$ is the output. We assume that we know an observer of the form

$$
\dot{\bar{x}}=\mathbf{f}(\bar{x})+\mathbf{k}(\bar{x}, y-\bar{y}) \quad \bar{y}=\mathbf{g}(\bar{x}),
$$

where $\bar{x} \in \mathbb{R}^{n_{x}}$ is the estimate of the state $x$ and $\bar{y} \in \mathbb{R}^{n_{y}}$ is the output to the observer. The observer (2) is designed such that $x-\bar{x}$ globally asymptotically converges to zero.

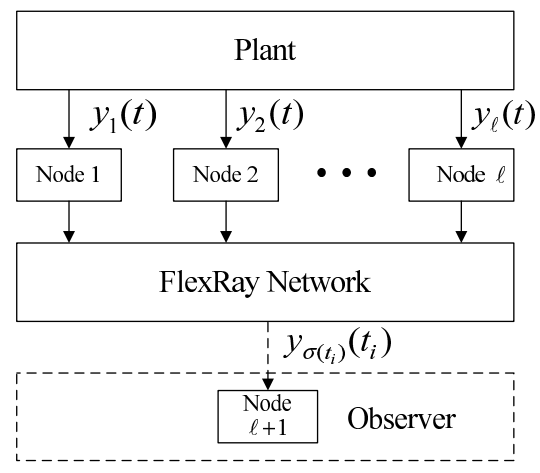

Fig. 1. Observer implemented on FlexRay

We consider the scenario where the plant and the observer communicate over a network and FlexRay is used to schedule transmissions. As illustrated in Figure 1, the measurements of the output $y$ are transmitted through $\ell \in \mathbb{Z}_{>0}$ nodes, where a node corresponds to a group of co-located sensors. We write $y=\left(y_{1}, \cdots, y_{\ell}\right)$ (after reordering, if necessary). Besides these $\ell$ sensor nodes, which can send and receive packets from the network, there are also some 'passive' nodes that only receive packets. Without loss of generality, we assume that there exists only one passive node where the observer is located, which corresponds to the node $\ell+1$ in Figure 1 .

Because of the network, the observer has no longer access to $y$, but to its networked version $\hat{y}$. At each transmission instant $t_{i}, i \in \mathbb{Z}_{>0}$, only one node is granted access to the channel by the scheduling policy to send its packet. The assignment of the transmission is specified by a piecewise function $\sigma: \mathbb{R}_{\geq 0} \rightarrow\{1, \cdots, \ell\}$. If the node $j \in\{1, \cdots, \ell\}$ is selected by the protocol at time $t_{i}, i \in \mathbb{Z}_{>0}, y_{\sigma\left(t_{i}\right)}\left(t_{i}\right)=$ $y_{j}\left(t_{i}\right)$ and then $\hat{y}$ satisfies

$$
\hat{y}_{j}\left(t_{i}^{+}\right)=y_{j}\left(t_{i}\right) \quad \hat{y}_{k}\left(t_{i}^{+}\right)=\hat{y}_{k}\left(t_{i}\right)
$$

for all $k \in\{1, \cdots, \ell\}$ satisfying $k \neq j$. During the intertransmission time intervals, for $i \in \mathbb{Z}_{>0}, \hat{y}$ evolves as

$$
\dot{\hat{y}}=\hat{\mathbf{f}}(\hat{y}, \bar{x}) \quad t \in\left[t_{i}, t_{i+1}\right],
$$

where $\hat{\mathbf{f}}$ denotes the holding function which is implemented at the observer to generate $\hat{y}$ between two successive transmission instants. When zero-order-hold devices are used, $\hat{\mathbf{f}}=0$. We allow for the moment the holding function to be general, as in (4), but we will show that this function has no impact on the result we present in Section III, which is different from $[9,10]$.
Our objective is to propose an emulation implementation method to preserve the convergence properties of the observer under the communication constraints induced by the network. We provide a brief description of FlexRay and specify the properties of the transmission instants sequence $\left\{t_{i}\right\}_{i \in \mathbb{Z}_{>0}}$ in the remaining part of this section.

\section{B. FlexRay}

We briefly present FlexRay and the assumptions we make on the network; further details can be found in Section III in [12]. FlexRay is characterized by pre-set communication cycles of length $T>0$, see [2]. Each cycle contains a static segment, a dynamic segment and two protocol segments called symbol window and network idle time, which are of respective lengths $T_{1}, T_{2}, T_{3}$ and $T_{4}>0$, as shown in Figure 2. The lengths of the protocol segments are often negligible with respect to the lengths of the static and dynamic segments, we therefore ignore these.

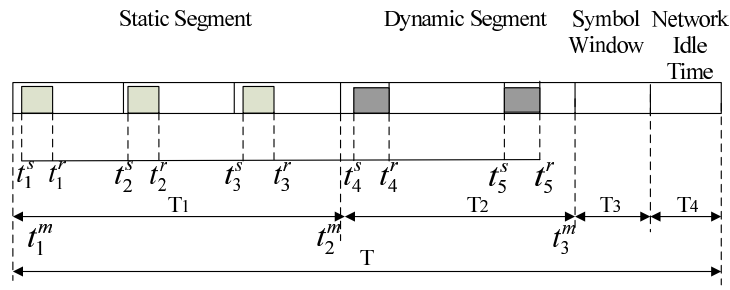

Fig. 2. FlexRay Communication Cycle [12]

Distinct network access techniques are applied for the static and the dynamic segments [2], [4], [8]. The static segment consists of time slots of equal length and it relies on a time division multiple access (TDMA) approach, where a single node is chosen by a static protocol and its packet is sent over the communication channel at the beginning of each time slot. The dynamic segment on the other hand employs the flexible time division multiple access (FTDMA) technique [1], which enables nodes to compete for accessing the network and a dynamic protocol can be implemented to select nodes to transmit messages based on the online information. The dynamic segment is composed of minislots, which are substantially shorter than static time slots and do not necessarily correspond to the transmission of a packet. The node with highest priority is assigned with the 'earliest' minislot and the minislots are idle when no nodes compete for access.

Let $t_{j}^{m}, j \in \mathbb{Z}_{>0}$ denote the segments switching instants. Let a packet be sent at transmission instant $t_{i}^{s}$ and received at a reception instant $t_{i}^{r}$ for $i \in \mathbb{Z}_{>0}$, as illustrated in Figure 2. For modeling purposes, we assume that FlexRay cycles start with the static segment without loss of generality and that the following properties hold.

\section{Assumption 1 ([12]):}

(a) The lengths of the symbol window and the network idle time are negligible, i.e. $T_{3}=T_{4}=0$.

(b) Data are transmitted instantaneously, i.e. $t_{i}:=t_{i}^{s}=t_{i}^{r}$ for all $i \in \mathbb{Z}_{>0}$. 
(c) Data coding time is negligible and a transmission occurs at the beginning of the two segments.

(d) The static time slots are of length $\tau_{\mathrm{MATI}, 1}>0$ and each inter-transmission time interval in the dynamic segments, denoted by $\tau_{d y, i}$, satisfies $\tau_{d y, i} \in\left[\varepsilon, \tau_{\mathrm{MATI}, 2}\right]$ for all $i \in \mathbb{Z}_{>0}$, where $\varepsilon>0$ refers to the length of a minislot.

Let $\tau_{\mathrm{MATI}, 1}>0$ be such that $T_{1}=N \tau_{\mathrm{MATI}, 1}$ for some ${ }^{1}$ $N \in \mathbb{Z}$. Under Assumption 1, it is shown in [12] that for any given $T_{1}, T_{2}>0$ the transmission instants sequence $\left\{t_{i}\right\}_{i \in \mathbb{Z}>0}$ satisfies

$$
\begin{aligned}
t_{i+1}-t_{i} & =\tau_{\text {MATI }, 1} & \forall t_{i}, t_{i+1} & \in\left[t_{2 n-1}^{m}, t_{2 n}^{m}\right] \\
t_{i+1}-t_{i} & \in\left[\varepsilon, \tau_{\text {MATI }, 2}\right] & \forall t_{i}, t_{i+1} & \in\left[t_{2 n}^{m}, t_{2 n+1}^{m}\right]
\end{aligned}
$$

where $n \in \mathbb{Z}_{>0}$ and the segment switching instants sequence $\left\{t_{j}^{m}\right\}_{j \in \mathbb{Z}>0}$ satisfies $t_{2 n}^{m}-1, t_{2 n-1}^{m}=T_{1}$ and $t_{2 n+1}^{m}-t_{2 n}^{m}=$ $T_{2}$.

\section{OBSERVER EMULATION}

We emulate the observer (2) as follows

$$
\dot{\bar{x}}=\mathbf{f}(\bar{x})+\mathbf{k}(\bar{x}, \hat{y}-\widetilde{y}) .
$$

The emulated observer (7) no longer depends on $y$, but on $\hat{y}$ because of the effect of the network. Furthermore, it does not depend on its own output $\bar{y}$ but on $\widetilde{y}$. The designed variable $\widetilde{y}$ can be interpreted as an artificially introduced networked version of $\bar{y}$ (see [11]), as it will be clear below.

Let $\widetilde{y}=\left(\widetilde{y}_{1}, \cdots, \widetilde{y}_{\ell}\right)$ and $\bar{y}=\left(\bar{y}_{1}, \cdots, \bar{y}_{\ell}\right)$. The dynamics of $\widetilde{y}$ is given by

$$
\begin{aligned}
& \dot{\tilde{y}}=\dot{\hat{y}} \quad t \in\left[t_{i}, t_{i+1}\right] \\
& \widetilde{y}_{j}\left(t_{i}^{+}\right)= \begin{cases}\bar{y}_{j}\left(t_{i}\right) & \text { if } \hat{y}_{j}\left(t_{i}^{+}\right)=y_{j}\left(t_{i}\right) \\
\widetilde{y}_{j}\left(t_{i}\right) & \text { otherwise. }\end{cases}
\end{aligned}
$$

In that way, $\widetilde{y}_{j}$ evolves along the same vector field as $\hat{y}_{j}$ between two successive transmission instants, and is reset to $\bar{y}_{j}$ when the corresponding node is updated.

Let $e_{0}:=\widetilde{y}-\bar{y} \in \mathbb{R}^{n_{e}}$ denote the (artificially introduced) network-induced error on the observer output, $e_{p}:=\hat{y}-$ $y \in \mathbb{R}^{n_{e}}$ be the network-induced error on the plant output, $n_{e}:=n_{y}$. In that way, (7) becomes

$$
\dot{\bar{x}}=\mathbf{f}(\bar{x})+\mathbf{k}(\bar{x}, y-\bar{y}-e) .
$$

where $e:=e_{o}-e_{p}$ is the network-induced error we study in the present paper.

Remark 1: If we implement (7) with $\bar{y}$ instead of $\widetilde{y}$, (9) turns into

$$
\dot{\bar{x}}=\mathbf{f}(\bar{x})+\mathbf{k}\left(\bar{x}, y-\bar{y}+e_{p}\right) .
$$

In this case, it is in general not possible to preserve the asymptotic convergence of the observer when zero-orderhold devices are used to generate $\hat{y}$, since the error $e_{p}$ is a priori non-vanishing as $\dot{e}_{p}=\dot{y}$ between two transmission instants and $\dot{y}$ is in general different from zero. Only a

\footnotetext{
${ }^{1}$ This comes from the fact that the time slots in the static segment are of equal length.
}

practical convergence may be guaranteed, see [9]. This issue is overcome with (7), as we show in Section VI.

Between the two successive transmission instants, the implementation of the observer leads to

$$
\begin{gathered}
\dot{e}=g_{e}(x, \bar{x}, e) \\
\dot{e}_{p}=g_{p}\left(x, \bar{x}, e_{p}\right)
\end{gathered} \quad t \in\left[t_{i}, t_{i+1}\right],
$$

where $i \in \mathbb{Z}_{>0}, g_{e}(x, \bar{x}, e):=\frac{\partial \mathbf{g}}{\partial x}(x) \mathbf{f}(x)-\frac{\partial \mathbf{g}}{\partial \bar{x}}(\bar{x})(\mathbf{f}(\bar{x})+$ $\mathbf{k}(\bar{x}, \mathbf{g}(x)-\mathbf{g}(\bar{x})-e))$ and $g_{p}\left(x, \bar{x}, e_{p}\right):=\hat{\mathbf{f}}(\mathbf{g}(x)+$ $\left.e_{p}, \bar{x}\right)-\frac{\partial \mathbf{g}}{\partial x}(x) \mathbf{f}(x)$. Note that the relevant network-induced error is $e$ not $e_{p}$ (in view of (9)) and the dynamics of $e$ during inter-transmission time intervals does not depend the holding functions $\hat{\mathbf{f}}$. Consequently, for the constructed $\widetilde{y}$ in (7) which determines the dynamics of $e$, different observer implementations make no difference to our main results. This is an important difference with [9], noting that the results in [9] can be viewed as a particular case of the present paper in the absence of perturbations.

The dynamics of the network-induced errors at transmission instants is governed by scheduling rules. We investigate this aspect in the following section.

\section{PRotocol MODEL}

\section{A. Model}

At the transmission instant $t_{i}, i \in \mathbb{Z}_{>0}$, if the node $j$ has accessed to the network, it holds that, in view of (3), (8) and the fact that $y\left(t_{i}^{+}\right)=y\left(t_{i}\right)$ and $\bar{y}\left(t_{i}^{+}\right)=\bar{y}\left(t_{i}\right)$ (since $y$ and $\bar{y}$ are not affected by transmissions),

$$
\begin{aligned}
& e_{p_{j}}\left(t_{i}^{+}\right)=\hat{y}_{j}\left(t_{i}^{+}\right)-y_{j}\left(t_{i}\right)=0 \\
& e_{o j}\left(t_{i}^{+}\right)=\widetilde{y}_{j}\left(t_{i}^{+}\right)-\bar{y}_{j}\left(t_{i}\right)=0
\end{aligned}
$$

for $j \in\{1, \cdots, \ell\}$, and

$$
\begin{aligned}
& e_{p_{k}}\left(t_{i}^{+}\right)=\hat{y}_{k}\left(t_{i}^{+}\right)-y_{k}\left(t_{i}\right)=e_{p_{k}}\left(t_{i}\right) \\
& e_{o k}\left(t_{i}^{+}\right)=\widetilde{y}_{k}\left(t_{i}^{+}\right)-\bar{y}_{k}\left(t_{i}\right)=e_{o k}\left(t_{i}\right)
\end{aligned}
$$

for $k \in\{1, \cdots, \ell\}$ satisfying $k \neq j$. Following the terminology of [7], we call the equation given below that specifies how $e_{p}$ at transmission times $t_{i}$ is mapped at $t_{i}^{+}$as the protocol equation

$$
e_{p}\left(t_{i}^{+}\right)=h_{p}\left(i, e\left(t_{i}\right), e_{p}\left(t_{i}\right), q\left(t_{i}\right)\right),
$$

where $q: \mathbb{R}_{\geq 0} \rightarrow\{1,2\}$ is a switching signal indicating which segment is active: $q(t)=1$ denotes the static segment and $q(t)=2$ represents the dynamic segment, for $t \geq 0$. The mapping $h_{p}$, which is allowed to depend on both $e_{p}$ and $e$, has the form of

$$
\begin{aligned}
& h_{p}\left(i, e\left(t_{i}\right), e_{p}\left(t_{i}\right), q\left(t_{i}\right)\right):=\left(2-q\left(t_{i}\right)\right) h_{p, 1}\left(i, e\left(t_{i}\right), e_{p}\left(t_{i}\right)\right) \\
& \quad+\left(q\left(t_{i}\right)-1\right) h_{p, 2}\left(i, e\left(t_{i}\right), e_{p}\left(t_{i}\right)\right),
\end{aligned}
$$

where $h_{p, 1}$ and $h_{p, 2}$ respectively denote the scheduling rule corresponding to the static and the dynamical segment.

On the other hand, considering the definition of $e$ and the case when the $j$-th node is selected, we have that

$$
\begin{aligned}
& e_{j}\left(t_{i}^{+}\right)=\left(\widetilde{y}_{j}\left(t_{i}^{+}\right)-\bar{y}_{j}\left(t_{i}^{+}\right)\right)-\left(\hat{y}_{j}\left(t_{i}^{+}\right)-y_{j}\left(t_{i}^{+}\right)\right)=0 \\
& e_{k}\left(t_{i}^{+}\right)=\left(\widetilde{y}_{k}\left(t_{i}^{+}\right)-\bar{y}_{k}\left(t_{i}^{+}\right)\right)-\left(\hat{y}_{k}\left(t_{i}^{+}\right)+y_{k}\left(t_{i}^{+}\right)\right)=e_{k}\left(t_{i}\right)
\end{aligned}
$$


hold for $j, k \in\{1, \cdots, \ell\}$ satisfying $k \neq j$. With the mapping $h_{e}$ to specify how $e$ evolves at each transmission instants, we write (15) as $e\left(t_{i}^{+}\right)=h_{e}\left(i, e\left(t_{i}\right), e_{p}\left(t_{i}\right), q\left(t_{i}\right)\right)$, which can also be formed as (14) with $h_{e, 1}$ and $h_{e, 2}$ denoting the scheduling rules corresponding to the static and dynamic segments. An example is given below on the construction of $h_{e}$.

In view of (11), (12) and (15), we see that the $e$-subsystem is strongly related with the $e_{p}$-subsystem at transmission instants. It is shown in Section VII of [11], that the $e$ subsystem inherits the stability property of the $e_{p}$-subsystem at jumps, under mild conditions. This will be important in Section VI when we study the stability of the overall system. We next present an example of protocols which are compatible with FlexRay.

\section{B. Example}

The RR protocol can be used to govern the static segment. It grants access to the nodes in a periodic fashion. In view of [7], $h_{p, 1}$ is defined by, for $e_{p} \in \mathbb{R}^{n_{e}}$ and $i \in \mathbb{Z}_{>0}$,

$$
h_{p, 1}\left(i, e_{p}\right):=(I-\Delta(i)) e_{p},
$$

where $\Delta(i)=\operatorname{diag}\left\{\tilde{\delta}_{1}(i) I_{n_{1}}, \cdots, \tilde{\delta}_{\ell}(i) I_{n_{\ell}}\right\}, I_{n_{s}}$ is the identity matrix of dimension $n_{s}$ with $\sum_{s=1}^{\ell} n_{s}=n_{e}, \tilde{\delta}_{s}$ satisfies $\tilde{\delta}_{s}(i)=1$ when $s-1=i \bmod \ell$ and $\tilde{\delta}_{s}(i)=0$ otherwise, for $s \in\{1, \ldots, \ell\}$ and $i \in \mathbb{Z}_{\geq 0}$.

We consider a TOD-like protocol for the dynamic segment, which grants access to the node where $\left|e_{j}\right|\left(\right.$ not $\left.\left|e_{p_{j}}\right|\right)$ is the biggest. Hence, the function $h_{p, 2}$ is defined as ([7])

$$
h_{p, 2}\left(e, e_{p}\right):=(I-\Psi(e)) e_{p}
$$

with $\Psi(e):=\operatorname{diag}\left\{\psi_{1}(e) I_{n_{1}}, \psi_{2}(e) I_{n_{2}}, \cdots, \psi_{\ell}(e) I_{n_{\ell}}\right\}$. The function $\psi_{s}$ satisfies $\psi_{s}(e)=1$ when $s=$ $\min \left(\arg \max _{j \in\{1, \cdots, \ell\}}\left|e_{j}\right|\right)$ and $\psi_{s}(e)=0$ otherwise, for $s \in\{1, \cdots, \ell\}$ and $i \in \mathbb{Z}_{>0}$.

Now, we derive the definitions of $h_{e, 1}$ and $h_{e, 2}$. From (11), (12) and (15), we see that the e-subsystem has the same dynamical property as the $e_{p}$-subsystem at transmission instants: its $j$-th component resets to zero and other components keep unchanged when the $j$-th node is chosen. Consequently, in view of (16) and (17), for any $e \in \mathbb{R}^{n_{e}}$

$$
\begin{aligned}
h_{e, 1}(i, e) & =(I-\Delta(i)) e \\
h_{e, 2}(e) & =(I-\Psi(e)) e,
\end{aligned}
$$

where $\Delta$ and $\Psi$ are defined above.

Remark 2: Note that the TOD protocol above requires the use of smart sensors that have sufficient computational capacities to run a copy of the observer located in the $(\ell+1)$ th node. That is, at each node $j \in\{1, \cdots, \ell+1\}$, an observer is run as

$$
\dot{\bar{x}}^{j}=\mathbf{f}\left(\bar{x}^{j}\right)+\mathbf{k}\left(\bar{x}, \hat{y}-\widetilde{y}^{j}\right)
$$

with $\widetilde{y}$ given in (8). They are synchronized, i.e. $\bar{x}^{j}(t)=$ $\bar{x}^{k}(t)$ for all $t$ and $j, k \in\{1, \cdots, \ell+1\}$, by assuming that $\bar{x}^{j}$ and $\bar{x}^{k}$ start with the same initial condition. Thanks to the assignment algorithm $\sigma$ (recall the algorithm of $\sigma$ above equation (3)), the available information of the $j$-th node for $j \in\{1, \cdots, \ell\}$ and $t \in\left[t_{i}, t_{i+1}\right)$ is given as below.

1) The $j$-th (sensor) node: $\sigma\left(t_{i}\right), y_{\sigma\left(t_{i}\right)}\left(t_{i}\right), y_{j}(t), \bar{x}^{j}(t)$.

2) The $(\ell+1)$-th (passive) node: $\sigma\left(t_{i}\right), y_{\sigma\left(t_{i}\right)}\left(t_{i}\right), \bar{x}^{\ell+1}(t)$. Since each node receives $y_{\sigma\left(t_{i}\right)}\left(t_{i}\right)$ and then the same input signal $\hat{y}$, and they are synchronized for all time (unless a computational glitch occurs) and the convergence property analysis reduces to studying a single observer.

\section{NCS MODEL}

We now implement the observer over the network and model the overall dynamics as a hybrid system using the formalism of [3]. We introduce for this purpose two clock variables $\tau_{1}, \tau_{2} \in \mathbb{R}_{\geq 0}$ like in [12]. The variable $\tau_{1}$ represents the time elapsed since the last transmission and $\tau_{2}$ denotes the time elapsed since the last segment switch. We also introduce $\kappa \in \mathbb{Z}_{\geq 0}$ to count the number of transmissions. Let $v:=(\xi, x), n_{v}:=n_{\xi}+n_{x}$ and $\varphi:=\left(e, e_{p}, \kappa, \tau_{1}, \tau_{2}, q\right)$, where $\xi:=x-\bar{x} \in \mathbb{R}^{n_{\xi}}$ with $n_{\xi}=n_{x}$ is the observation error. Then, the following hybrid system is obtained.

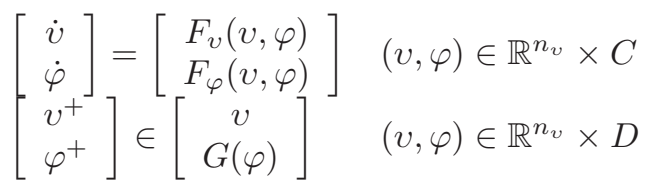

where $C:=\mathbb{R}^{2 n_{e}} \times \mathbb{Z}_{\geq 0} \times\left(C_{1} \cup C_{2}\right), D:=\mathbb{R}^{2 n_{e}} \times \mathbb{Z}_{\geq 0} \times$ $\left(D_{1} \cup D_{2}\right)$ with $C_{1}:=\left[0, \tau_{\mathrm{MATI}, 1}\right] \times\left[0, T_{1}\right] \times\{1\}, C_{2}:=$ $\left[0, \tau_{\text {MATI }, 2}\right] \times\left[0, T_{2}\right] \times\{2\}, D_{1}:=\left(\left(\left\{\tau_{\text {MATI }, 1}\right\} \times\left[0, T_{1}\right]\right) \cup\right.$ $\left.\left(\left[\varepsilon, \tau_{\text {MATI }, 2}\right] \times\{0\}\right) \cup\left(\left[0, \tau_{\text {MATI }, 1}\right] \times\left\{T_{1}\right\}\right)\right) \times\{1\}$ and $D_{2}:=\left(\left(\left[\varepsilon, \tau_{\text {MATI }, 2}\right] \times\left[0, T_{2}\right]\right) \cup\left(\left\{\tau_{\text {MATI }, 1}\right\} \times\{0\}\right) \cup\right.$ $\left.\left([0, \varepsilon] \times\left\{T_{2}\right\}\right)\right) \times\{2\}$.

The flow set $C$ and the jump set $D$ are defined based on the conditions of Assumption 1. In particular, the definition of $C$ means that system (19) is allowed to flow in the static segment $(q=1)$ when $\tau_{1} \leq \tau_{\text {MATI,1 }}$ and in the dynamic segment $(q=2)$ when $\tau_{1} \leq \tau_{\text {MATI, } 2}$. The system experiences a jump, which corresponds to a transmission when $\tau_{1}=\tau_{\text {MATI, } 1}$ and $\tau_{2} \leq T_{1}$ for $q=1$, and when $\tau_{1} \in\left[\varepsilon, \tau_{\mathrm{MATI}, 2}\right]$ and $\tau_{2} \leq T_{2}$ for $q=2$. The other kind of jumps correspond to the segment switches, which happen at $\tau_{2}=T_{1}$ when $q=1$ and $\tau_{2}=T_{2}$ when $q=2$. The transmissions are assumed to occur at the beginning of the two segments, i.e. when $\tau_{1} \in\left[\varepsilon, \tau_{\text {MATI,2 }}\right]$ at the beginning of the static segments, where the lower bound $\varepsilon$ is the minislot length (see item (d) of Assumption 1), and when $\tau_{1}=\tau_{\text {MATI, } 1}$ at the beginning of the dynamic segment.

The flow mappings $F_{v}$ and $F_{\varphi}$ have the following form $F_{v}(v, \varphi):=\left[f_{\xi}^{T}(v, e) \quad f_{x}^{T}(v, e)\right]^{T}, F_{\varphi}(v, \varphi):=$ $\left[\begin{array}{llllll}g_{e}^{T}(v, e) & g_{p}^{T}\left(v, e_{p}\right) & 0 & 1 & 1 & 0\end{array}\right]^{T}$. The vector fields $g_{e}$ and $g_{p}$ come from (10) and the expressions of $f_{\xi}$ and $f_{z}$ are obtained by direct calculations from (1) and (2). Note that, thanks to (7), the continuous vector fields $g_{e}, f_{\xi}$ and $f_{z}$ are independent to the variable $e_{p}$. The jump mapping $G$ is defined as

$$
G(\varphi):= \begin{cases}G_{1}(\varphi) & \varphi \in \Omega_{1} \\ G_{2}(\varphi) & \varphi \in \Omega_{2} \\ \left\{G_{1}(\varphi), G_{2}(\varphi)\right\} & \varphi \in \Omega_{3}\end{cases}
$$


with $G_{1}(\varphi):=\left[h_{e}^{T}\left(\kappa, e, e_{p}, q\right) h_{p}^{T}\left(\kappa, e, e_{p}, q\right) \kappa+10 \tau_{2} q\right]^{T}$, $G_{2}(\varphi):=\left[\begin{array}{llllll}e^{T} & e_{p}^{T} & \kappa & \tau_{1} & 0 & 3-q\end{array}\right]^{T}$ and $\Omega_{i}:=$ $\mathbb{R}^{2 n_{e}} \times \mathbb{Z}_{\geq 0} \times \bar{\Omega}_{i}$, where $\bar{\Omega}_{i}$ for $i \in\{1,2,3\}$ are given by

$\begin{aligned} & \bar{\Omega}_{1}:=\left(\left(\left(\left\{\tau_{\text {MATI }, 1}\right\} \times\left[0, T_{1}\right)\right) \cup\left(\left[\varepsilon, \tau_{\text {MATI }, 2}\right] \times\{0\}\right)\right) \times\{1\}\right) \\ & \cup\left(\left(\left(\left[\varepsilon, \tau_{\text {MATI }, 2}\right] \times\left[0, T_{2}\right)\right) \cup\left(\left\{\tau_{\text {MATI }, 1}\right\} \times\{0\}\right)\right) \times\{2\}\right) \\ & \bar{\Omega}_{2}:=\left(\left[0, \tau_{\text {MATI }, 1}\right) \times\left\{T_{1}\right\} \times\{1\}\right) \cup\left([0, \varepsilon) \times\left\{T_{2}\right\} \times\{2\}\right) \\ & \bar{\Omega}_{3}:=\left(\left\{\tau_{\text {MATI }, 1}\right\} \times\left\{T_{1}\right\} \times\{1\}\right) \cup\left(\left[\varepsilon, \tau_{\text {MATI }, 2}\right] \times\left\{T_{2}\right\} \times\{2\}\right) .\end{aligned}$

The jump map $G$ is defined on the set $D\left(\Omega_{1} \cup \Omega_{2} \cup \Omega_{3}=D\right)$ and is OSC. Hence, noting that $G$ is locally bounded relative to $D$, flow maps $F_{v}$ and $F_{\varphi}$ are continuous and the sets $C$ and $D$ are closed, we have that the hybrid system (19) is well-posed, see Chapter 6 in [3] for more detail.

\section{MAIN RESUltS}

We assume that the scheduling rules which govern the transmissions of the static and the dynamic segments satisfy the properties listed below.

Assumption 2: For each $m \in\{1,2\}$, there exist $W_{m}$ : $\mathbb{R}^{n_{e}} \times \mathbb{Z}_{\geq 0} \rightarrow \mathbb{R}_{\geq 0}$, which is locally Lipschitz in its first argument, a continuous function $H_{m}: \mathbb{R}^{n_{v}} \rightarrow \mathbb{R}_{\geq 0}$, constants $\underline{\alpha}_{W_{m}}, \bar{\alpha}_{W_{m}}>0, L_{m} \geq 0$ and $\rho_{m} \in(0,1)$ such that for any $\kappa \in \mathbb{Z}_{\geq 0}$ and $e, e_{p} \in \mathbb{R}^{n_{e}}$

1) $\underline{\alpha}_{W_{m}}|e| \leq W_{m}(e, \kappa) \leq \bar{\alpha}_{W_{m}}|e|$,

2) $W_{m}\left(h_{e}\left(\kappa, e, e_{p}, m\right), \kappa+1\right) \leq \rho_{m} W_{m}(e, \kappa)$, and for all $v \in \mathbb{R}^{n_{v}}, \kappa \in \mathbb{Z}_{\geq 0}$ and almost all $e \in \mathbb{R}^{n_{e}}$

3) $\left\langle\frac{\partial W_{m}(e, \kappa)}{\partial e}, g_{e}(v, e)\right\rangle \leq L_{m} W_{m}(e, \kappa)+H_{m}(v)$.

Items 1)-2) of Assumption 2 mean that the $e$-subsystem at jumps is UGES. Item 3 ) implies that an exponential growth condition of the $e$-subsystem holds during two consecutive transmissions. Using the same technique as Section VII of [11], we can show that items 1)-2) of Assumption 2 are valid for example for the RR-TOD protocol in Section IV-B. Item 3 ) of Assumption 2 is verified when $g_{e}$ is globally Lipschitz, vanishes at the origin and $\frac{\partial W}{\partial e}$ is bounded, which is the case for RR and TOD protocols in view of Section V in [7]. We next assume that the observer has been designed such that the following conditions hold.

Assumption 3: There exist a continuously differentiable function $V: \mathbb{R}^{n_{\xi}} \rightarrow \mathbb{R}_{\geq 0}$ and $\underline{\alpha}_{V}, \bar{\alpha}_{V} \in \mathcal{K}_{\infty}$ such that for each $m \in\{1,2\}$ there exist a positive definite continuous function $\varrho_{m}$ and $\gamma_{m} \geq 0$ such that for all $v \in \mathbb{R}^{n_{v}}, e \in \mathbb{R}^{n_{e}}$ and $\kappa \in \mathbb{Z}_{\geq 0}$,

1) $\underline{\alpha}_{V}(|\xi|) \leq V(\xi) \leq \bar{\alpha}_{V}(|\xi|)$,

2) $\left\langle\nabla V(\xi), f_{\xi}(v, e)\right\rangle \leq-\varrho_{m}(|\xi|)-\varrho_{m}(|e|)-H_{m}^{2}(\xi)+$ $\gamma_{m}^{2} W_{m}^{2}(e, \kappa)$, where the functions $W_{m}$ and $H_{m}$ come from Assumption 2.

We show later in Section VII that these conditions are satisfied by a class of high-gain observers.

We next provide explicit bounds on the constants $\tau_{\text {MATI,1 }}$ and $\tau_{\text {MATI,2 }}$. We first introduce some notations for the ease of presentation. Let $\rho_{m} \in(0,1)$ and $\gamma_{m}>0$ come from Assumptions 2 and $3, \pi_{m}:=\bar{\alpha}_{W_{3-m}} / \underline{\alpha}_{W_{m}}$ and

$$
c_{m}(s):=\frac{\gamma_{m}}{\gamma_{m} / k_{m}-d_{m}(s)}+\Delta_{m}+\frac{1}{\rho_{m} s},
$$

where $\Delta_{m}:=\frac{(M-1) \gamma_{m}}{\rho_{m}^{2}}, d_{m}(s):=\frac{k_{m}-\gamma_{m} \rho_{m}}{k_{m} \rho_{m} \exp \left(k_{m} \rho_{m} s\right)}$ for $s \geq$ 0 with $M:=\max \left\{1, \frac{\gamma_{2} \rho_{1} \rho_{2}}{\gamma_{1} \pi_{2}^{2}}, \frac{\gamma_{1} \rho_{1} \rho_{2}}{\gamma_{2} \pi_{1}^{2}}\right\}$ and $k_{m}:=\max \left\{\gamma_{m}\right.$, $\left.\frac{\gamma_{m}^{2} \pi_{3-m}^{2}}{\gamma_{3-m} \rho_{m} \rho_{3-m}}\right\}$. Note that $c_{m}(s)$ is strictly positive since it decreases when $s$ grows and $c_{m}(s) \rightarrow k_{m}+\Delta_{m}>0$ when $s \rightarrow \infty$.

Assumption 4: For each $T_{1}, T_{2}>0$,

$$
\tau_{\mathrm{MATI}, 1}=\frac{T_{1}}{\left\lceil T_{1} / \bar{\tau}_{\mathrm{MATI}, 1}\right\rceil} \tau_{\mathrm{MATI}, 2}=\bar{\tau}_{\mathrm{MATI}, 2},
$$

where, for $m \in\{1,2\}$,

$$
\begin{aligned}
& \bar{\tau}_{\text {MATI }, m} \\
& \quad= \begin{cases}\frac{1}{L_{m} r_{m}\left(T_{m}\right)} \arctan \left(\lambda\left(T_{m}\right)\right) & \sqrt{\gamma_{m} c_{m}\left(T_{m}\right)}>L_{m} \\
\frac{\rho_{m}}{\rho_{m} \gamma_{m}+L_{m}}-\frac{1}{\rho_{m} L_{m}+\gamma_{m}} & \sqrt{\gamma_{m} c_{m}\left(T_{m}\right)}=L_{m} \\
\frac{1}{L_{m} r_{m}\left(T_{m}\right)} \operatorname{arctanh}\left(\lambda\left(T_{m}\right)\right) & \sqrt{\gamma_{m} c_{m}\left(T_{m}\right)}<L_{m},\end{cases}
\end{aligned}
$$

with $L_{m}, \gamma_{m}>0$ and $\rho_{m} \in(0,1)$ coming from Assumptions 2 and $3, \lambda(s):=\frac{r_{m}(s)\left(1-\rho_{m}\right)}{2 \frac{\rho_{m}}{1+\rho_{m}}\left(\frac{\gamma_{m}}{L_{m}}-1\right)+1+\rho_{m}}, r_{m}(s):=$ $\sqrt{\left|\frac{\gamma_{m} c_{m}(s)}{L_{m}^{2}}-1\right|}$ and $c_{m}: \mathbb{R}_{>0} \rightarrow \mathbb{R}_{>0}$ given by (22).

Recall that the time slots in the static segment are of equal length. The first equation in (23) is justified since $T_{1}$ has to be a multiple of $\tau_{\text {MATI,1 }}$ (see Section II-B). Compared with the MATI in Section VIII in [11], where the observer design problem is considered for NCS scheduled by non-switched protocols, the MATIs in (23) adapt to the segment lengths and the latter can be arbitrarily selected. The smaller $T_{m}$ the smaller $\tau_{\mathrm{MATI}, m}$. On the other hand, when $T_{1}$ goes to infinity, which corresponds to the case where there is only one segment, we recover the MATI bounds in [11] by taking $M=1$ and $k_{1}=\gamma_{1}$, which is possible since we can choose parameters $\gamma_{2}, \pi_{1}, \pi_{2}, \rho_{2}$ in an arbitrary way as the segment $q=2$ will never be activated.

For the ease of presentation, we write system (19) as

$$
\begin{array}{ll}
\dot{\zeta}=\mathcal{F}(\zeta) & \zeta \in \mathcal{C} \\
\zeta^{+} \in \mathcal{G}(\zeta) & \zeta \in \mathcal{D}
\end{array}
$$

where $\zeta:=(v, \varphi), \mathcal{C}:=\mathbb{R}^{n_{v}} \times C, \mathcal{D}:=\mathbb{R}^{n_{v}} \times D, \mathcal{F}(\zeta):=$ $\left(F_{v}(v, \varphi), F_{\varphi}(v, \varphi)\right)$ and $\mathcal{G}(\zeta):=(v, G(\varphi))$.

It should be emphasized that, because we implement the observer as in (7) and because we have selected the coordinates in (19), we can apply the results of [12] to study the stability of system (25). In particular, we can construct the hybrid Lyapunov function below

$$
\begin{gathered}
U(\zeta):=V(\xi)+\gamma_{1} \phi_{1}\left(\tau_{1}\right) \theta_{1}\left(\tau_{2}\right) W_{1}^{2}(e, \kappa)(2-q) \\
+\gamma_{2} \phi_{2}\left(\tau_{1}\right) \theta_{2}\left(\tau_{2}\right) W_{2}^{2}(e, \kappa)(q-1),
\end{gathered}
$$

where $\theta_{1}, \theta_{2}, \phi_{1}$ and $\phi_{2}$ are appropriately designed functions such that $U$ is positive definite on the set $\mathcal{C} \cup \mathcal{D}$, decreases during flow and does not increase at jumps, see [12] for more 
details. With such $U$, we can derive the following result. The proof is omitted as it follows from Proposition 1 and Theorem 1 in [12].

Theorem 1: Suppose that Assumptions 2-4 hold for system (25) and let $\mathcal{A}:=\{\zeta: \xi=0, e=0\}$. Then, there exists $\beta \in \mathcal{K} \mathcal{L}$ such that all solutions $\zeta$ to system (25) satisfy

$$
|\zeta(t, j)|_{\mathcal{A}} \leq \beta\left(|\zeta(0,0)|_{\mathcal{A}}, t+j\right), \quad \forall(t, j) \in \operatorname{dom} \zeta .
$$

\section{CASE STUDY}

Consider the nonlinear system

$$
\dot{x}=A x+\eta(x), \quad y=C x
$$

where $x \in \mathbb{R}^{n_{x}}, y \in \mathbb{R}^{n_{y}}, A, B$ and $C$ are constant matrices of appropriate dimensions, and the vector field $\eta: \mathbb{R}^{n_{x}} \rightarrow$ $\mathbb{R}^{n_{x}}$ is globally Lipschitz with a Lipschitz constant $\bar{M} \geq 0$. We consider an observer of the form

$$
\dot{\bar{x}}=A \bar{x}+\eta(\bar{x})+\mathbf{k}(y-\bar{y}), \quad \bar{y}=C \bar{x}
$$

where $\mathbf{k}$ is a matrix to be designed. Suppose that the measurements of $y$ are transmitted to the network through $\ell$ nodes for some $\ell \in \mathbb{Z}_{>0}$ and the matrix $\mathbf{k}$ is such that the following condition holds.

Assumption 5: There exists a real symmetric positive definite matrix $P$ such that $V: \xi \mapsto \xi^{T} P \xi$ and $\frac{\partial V}{\partial \xi}((A-$ $\mathrm{k} C) \xi+\eta(x)-\eta(x-\xi)) \leq-c V$ for all $\xi, x \in \mathbb{R}^{n_{x}}$ and some $c>\frac{\max \left\{a_{1}^{2}, a_{2}^{2}\right\}}{\lambda_{\min }(P)}$, where $\xi:=x-\bar{x}, a_{1}:=$ $\sqrt{\ell}(|C(A-\mathbf{k} C)|+\bar{M}|C|), a_{2}:=|C(A-\mathbf{k} C)|+\bar{M}|C| \cdot \square$

Assumption 5 holds for any detectable linear time invariant systems and is satisfied by the high-gain observers in [5]. We now implement the observer (29) over FlexRay as explained in Section III with the transmissions being scheduled by the RR-TOD protocol presented in Section IV-B. The emulated observer has form of

$$
\dot{\bar{x}}=A \bar{x}+\eta(\bar{x})+\mathbf{k}(\hat{y}-\widetilde{y})
$$

with $\widetilde{y}$ being defined as (8). We can rewrite the obtained system into (19) with $h_{e}, h_{p}$ defined by (16)-(18), and, for any $\xi, x \in \mathbb{R}^{n_{x}}$ and $e \in \mathbb{R}^{n_{y}}$,

$$
\begin{aligned}
& f_{\xi}(\xi, x, e):=(A-\mathbf{k} C) \xi+\eta(x)-\eta(x-\xi)+\mathbf{k} e \\
& f_{x}(\xi, x, e):=A x+\eta(x) \\
& g_{e}(\xi, x, e):=C(A-\mathbf{k} C) \xi+C(\eta(x)-\eta(x-\xi))+C \mathbf{k} e \\
& g_{p}\left(\xi, x, e_{p}\right):=\hat{\mathbf{f}}\left(C x+e_{p}, x-\xi\right)-C(A x+\eta(x)) .
\end{aligned}
$$

We next verify Assumptions 2 and 3 provided that Assumption 5 holds. We present the conclusion in the following proposition. The proof is omitted due to space limitations.

Proposition 1: Consider system (19) with $h_{e}, h_{p}$ defined by (16)-(18), $f_{\xi}, f_{x}, g_{x}$ and $g_{p}$ given above, and suppose Assumption 5 holds. Then, there exist $W_{1}$ and $W_{2}: \mathbb{R}^{n_{y}} \times$ $\mathbb{Z}_{\geq 0} \rightarrow \mathbb{R}_{\geq 0}$ such that Assumptions 2 and 3 hold with the $V: \mathbb{R}^{n_{x}} \rightarrow \mathbb{R}_{\geq 0}$ defined as Assumption 5, constants $\underline{\alpha}_{W_{1}}=1, \bar{\alpha}_{W_{1}}=\sqrt{\ell}, \underline{\alpha}_{W_{2}}=\bar{\alpha}_{W_{2}}=1, \rho_{1}=\rho_{2}=$ $\sqrt{\frac{\ell-1}{\ell}}, L_{1}=\sqrt{\ell}|C \mathbf{k}|$ for $H_{1}(\xi):=a_{1}|\xi|, L_{2}=|C \mathbf{k}|$ for
$H_{2}(\xi):=a_{2}|\xi|$ for all $\xi \in \mathbb{R}^{n_{x}}, \gamma_{1}=\sqrt{\frac{2}{b_{1}}|P \mathbf{k}|^{2}+\nu}$ and $\gamma_{2}=\sqrt{\frac{2}{b_{2}}|P \mathbf{k}|^{2}+\nu}$ for an arbitrary $\nu>0$, where $a_{1}$ and $a_{2} \geq 0$ come from Assumption 5 .

Note that all parameters needed to calculate MATI bounds $\tau_{\text {MATI,1 }}$ and $\tau_{\text {MATI,2 }}$ are given in Proposition 1 . For any given length $T_{1}$ and $T_{2}$, we can calculate $\tau_{\mathrm{MATI}, 1}$ and $\tau_{\text {MATI,2 }}$ using Assumption 4 and Theorem 1 shows that the observation error $\xi$ and the network induced error $e$ globally asymptotically converges to the origin.

\section{CONCLUSION}

We have proposed a method to design emulated observer for nonlinear networked control systems with FlexRay. We have generalized prior results on the observer emulation for NCS in [11] to be applicable for FlexRay networks. A novel hybrid model has been proposed for that purpose, with which we showed that the observer convergence to zero is ensured provided that, for each segment, the scheduling rules are uniformly globally exponentially stable and the maximal allowable transmission interval satisfy given explicit bounds. The analysis relies on the use of an hybrid Lyapunov function we recently constructed to investigate the stabilization of NCS with FlexRay. In future work, we will consider the case where the measurements are noisy and where the plant dynamics depend on some external inputs.

\section{REFERENCES}

[1] G. Cena and A. Valenzano. On the properties of the flexible time division multiple access technique. IEEE Transactions on Industrial Informatics, 2:86-94, 2006.

[2] Flexray Consortium. FlexRay Communications System - Protocol Specification V2.1 Rev.A, 2005.

[3] R. Goebel, R. G. Sanfelice, and A. R. Teel. Hybrid dynamical systems: Modeling, Stability, and Robustness. Princeton University Press, 2012.

[4] Australia National Instruments. FlexRay Automotive Communication Bus Overview, 2009.

[5] H. K. Khalil and L. Praly. High-gain observers in nonlinear feedback control. International Journal of Robust and Nonlinear Control, 24:993-1015, 2013.

[6] P. Naghshtabrizi and J. P. Hespanha. Analysis of distributed control systems with shared communication and computation resources. In Proceedings of the American Control Conference, pages 3384-3389, Saint Louis, USA, 2009.

[7] D. Nešić and A. R. Teel. Input-output stability properties of networked control systems. IEEE Transactions on Automatic Control, 49:16501667, 2004.

[8] T. Pop, P. Pop, P. Eles, Z. Peng, and A. Andrei. Timing analysis of the Flexray communication protocol. Real-Time Systems, 39:205-235, 2008.

[9] R. Postoyan and D. Nešić. A framework for the observer design for networked control systems. IEEE Transactions on Automatic Control, 57:1309-1314, 2012.

[10] R. Postoyan and D. Nešić. On emulated nonlinear reduced-order observers for networked control systems. Automatica, 48:645-652, 2012.

[11] R. Postoyan, N. van de Wouw, D. Nešić, and W.P.M.H. Heemels. Tracking control for nonlinear networked control systems. IEEE Transactions on Automatic Control, 59:1539-1554, 2014.

[12] W. Wang, D. Nešić, and R. Postoyan. Emulation-based stabilization of networked control systems implemented on flexray. The 53th IEEE Conference on Decision and Control, Los Angeles, USA, 2014. 